\title{
EFFECT OF INITIAL STRESSES ON INCIDENT $q S V$-WAVES IN PRE-STRESSED ELASTIC HALF-SPACES
}

\author{
S. S. SINGH ${ }^{1}$
}

(Received 25 March, 2011; revised 5 October, 2011)

\begin{abstract}
The effect of initial stresses on incident quasi $S V$-waves at a plane interface between two dissimilar pre-stressed elastic half-spaces is investigated. The reflection and refraction coefficients of the reflected and refracted $q S V$ - and $q P$-waves are derived with the help of appropriate boundary conditions. The coefficients are found to be functions of the angle of incidence and the initial stresses and incremental elastic parameters of the prestressed elastic half-spaces.
\end{abstract}

2010 Mathematics subject classification: primary 74B05; secondary 74B10.

Keywords and phrases: $q S V$-wave, $q P$-wave, reflection and refraction coefficients, prestressed elastic half-space.

\section{Introduction}

The subject of elastic wave propagation has long been a matter of common interest in the fields of engineering, geophysics and seismology. Studies in this area give us valuable information about the media through which the waves travel. In particular, the seismic signals propagating through different layers of the Earth not only provide information about the internal structure of the Earth, but also about buried materials like minerals, crystals and metals. Keeping in view the importance of wave propagation, many researchers have investigated a number of problems related to reflection and refraction of elastic waves [1-4, 8-11, 15, 20-22, 24].

Pioneering work in developing the theory of waves in elastic media with initial stresses was done by Biot $[5,6]$, who developed the constitutive relations for a prestressed elastic medium and presented the equations of motion for elastic waves. Ogden and Sotiropoulos [14] studied the problem of interfacial waves along the plane boundary between a pre-stressed incompressible elastic solid half-space and a pre-stressed incompressible elastic solid layer of uniform thickness, obtaining the dispersion relation and the conditions on the pre-strain, pre-stress and material

\footnotetext{
${ }^{1}$ Mizoram University, Pachhunga University College, Department of Mathematics, Aizawl - 796001 , Mizoram, India; e-mail: saratcha32@yahoo.co.uk.

(c) Australian Mathematical Society 2012, Serial-fee code 1446-1811/2012 \$16.00
} 
parameters that ensure the existence of a unique interfacial wave speed at low and high frequencies. Khurana and Vashisth [12] analysed the problem of Love wave propagation in a pre-stressed elastic layer over a pre-stressed poroelastic solid halfspace. Chattopadhyay et al. [7] analysed the problem of wave propagation in a prestressed elastic medium and discussed the reflection of $P$ - and $S V$-waves at a free surface of an initially stressed elastic half-space. Norris [13] studied the problem of the propagation of plane waves in a pre-stressed elastic medium and showed that the pure longitudinal and shear waves can propagate only in certain specific directions. Sidhu and Singh [16] commented on the paper of Chattopadhyay et al. [7] and said that their results are not acceptable because the method of potentials used in their paper is not applicable for pre-stressed media. Later, Sidhu and Singh [17] investigated the problem of plane wave propagation in a pre-stressed elastic solid with incremental elastic coefficients possessing orthotropic symmetry. They obtained two types of plane waves, the so-called quasi $P$-waves and quasi $S V$-waves, showed that their velocities depend on the angle of propagation and derived the reflection coefficients of the reflected $P$ - and $S V$-waves. Sidhu and Singh [18] also obtained a condition on the incremental elastic constants for the existence of real values of the phase velocity of quasi $S V$-waves in a pre-stressed elastic solid. Recently, Singh and Tomar [23] studied the problem of $q P$-waves at a corrugated interface between two dissimilar pre-stressed elastic half-spaces, obtaining the reflection and refraction coefficients corresponding to regular and irregular waves using Rayleigh's method of approximation. Singh [19] attempted the problem of wave propagation in a pre-stressed piezoelectric half-space and discussed the effect of initial stresses on the reflection coefficients of the reflected $q P$ - and $q S V$-waves.

In this paper, the reflection and refraction of elastic waves due to incident $q S V$ waves at a plane interface between two dissimilar pre-stressed elastic half-spaces is investigated. The reflection and refraction coefficients of the reflected and refracted elastic waves are presented in closed form. These coefficients are obtained numerically for a particular model, and the results depicted graphically. The results of Sidhu and Singh [17] are recovered as a particular case of the present problem.

\section{Equations of motion}

Let us consider the Cartesian coordinates where the $x$-axis and $z$-axis are perpendicular to each other in the horizontal plane and the $y$-axis is vertical with its positive direction pointing downward. Consider two homogeneous pre-stressed elastic half-spaces, $M: 0 \leq y<\infty$ and $M^{\prime}:-\infty<y \leq 0$, separated by a plane interface, $y=0$. The parameters corresponding to the half-space $M^{\prime}$ are denoted with a prime, and those corresponding to $M$ without a prime. These half-spaces are either isotropic in finite strain or anisotropic with orthotropic symmetry. Isotropy in finite strain of a prestressed medium means that the stress is related to the finite strain by relations which are independent of the orientation of the stress field. The elastic medium is believed to be isotropic in the vicinity of the unstressed state but can be thought of as becoming anisotropic when finite strains are applied. 
The coordinate system is chosen to coincide with the principal directions of the initial stress, which are represented by its three principal components. Since the medium is isotropic in finite strain, the principal directions of the initial stress define three planes of symmetry for the incremental elastic properties. In this condition, the incremental stress-strain relations must possess orthotropic symmetry. The initial stress is defined by the principal components $S_{11}, S_{22}$ and $S_{33}$ in the half-space $M$, and $S_{11}^{\prime}, S_{22}^{\prime}$ and $S_{33}^{\prime}$ in $M^{\prime}$. If we consider the plane strain parallel to the $x, y$-plane with displacement components $U$ and $U^{\prime}$ in the $x$ direction and $V$ and $V^{\prime}$ in the $y$ direction, the third principal stresses $S_{33}$ and $S_{33}^{\prime}$ do not enter explicitly into the equations of motion. The equations of motion for $q S V$-wave propagation in the half-space $M$ are given by Biot [6] as

$$
\begin{aligned}
& B_{11} \frac{\partial^{2} U}{\partial x^{2}}+A_{3} \frac{\partial^{2} V}{\partial x \partial y}+A_{1} \frac{\partial^{2} U}{\partial y^{2}}=\rho \frac{\partial^{2} U}{\partial t^{2}} \\
& B_{22} \frac{\partial^{2} V}{\partial y^{2}}+A_{3} \frac{\partial^{2} U}{\partial x \partial y}+A_{2} \frac{\partial^{2} V}{\partial x^{2}}=\rho \frac{\partial^{2} V}{\partial t^{2}}
\end{aligned}
$$

where

$$
\begin{gathered}
A_{1}=Q+P / 2, \quad A_{2}=Q-P / 2, \quad A_{3}=B_{12}+A_{2}, \\
B_{12}=B_{21}+P, \quad P=S_{22}-S_{11} ;
\end{gathered}
$$

$\rho$ is the density and $B_{11}, B_{22}, B_{12}$ and $Q$ are the incremental elastic coefficients of $M$.

Similarly, the equations of motion for $q S V$-waves in $M^{\prime}$ are

$$
\begin{aligned}
& B_{11}^{\prime} \frac{\partial^{2} U^{\prime}}{\partial x^{2}}+A_{3}^{\prime} \frac{\partial^{2} V^{\prime}}{\partial x \partial y}+A_{1}^{\prime} \frac{\partial^{2} U^{\prime}}{\partial y^{2}}=\rho^{\prime} \frac{\partial^{2} U^{\prime}}{\partial t^{2}} \\
& B_{22}^{\prime} \frac{\partial^{2} V^{\prime}}{\partial y^{2}}+A_{3}^{\prime} \frac{\partial^{2} U^{\prime}}{\partial x \partial y}+A_{2}^{\prime} \frac{\partial^{2} V^{\prime}}{\partial x^{2}}=\rho^{\prime} \frac{\partial^{2} V^{\prime}}{\partial t^{2}}
\end{aligned}
$$

where

$$
\begin{gathered}
A_{1}^{\prime}=Q^{\prime}+P^{\prime} / 2, \quad A_{2}^{\prime}=Q^{\prime}-P^{\prime} / 2, \quad A_{3}^{\prime}=B_{12}^{\prime}+A_{2}^{\prime}, \\
B_{12}^{\prime}=B_{21}^{\prime}+P^{\prime}, \quad P^{\prime}=S_{22}^{\prime}-S_{11}^{\prime} ;
\end{gathered}
$$

$\rho^{\prime}$ is the density and $B_{11}^{\prime}, B_{22}^{\prime}, B_{12}^{\prime}$ and $Q^{\prime}$ are the incremental elastic coefficients of $M^{\prime}$.

\section{Wave propagation}

Consider a plane $q S V$-wave propagating through the half-space $M$ with phase velocity $c_{2}$ incident to the plane interface making an angle $\theta_{0}$ with the normal. Sidhu and Singh [17] showed that $c_{2}$ is given by

$$
2 \rho_{1} c_{2}^{2}=E_{1}\left(\theta_{0}\right)+E_{2}\left(\theta_{0}\right)-\sqrt{\left(E_{1}\left(\theta_{0}\right)-E_{2}\left(\theta_{0}\right)\right)^{2}+4 A_{3}^{2} \sin ^{2} \theta_{0} \cos ^{2} \theta_{0}},
$$




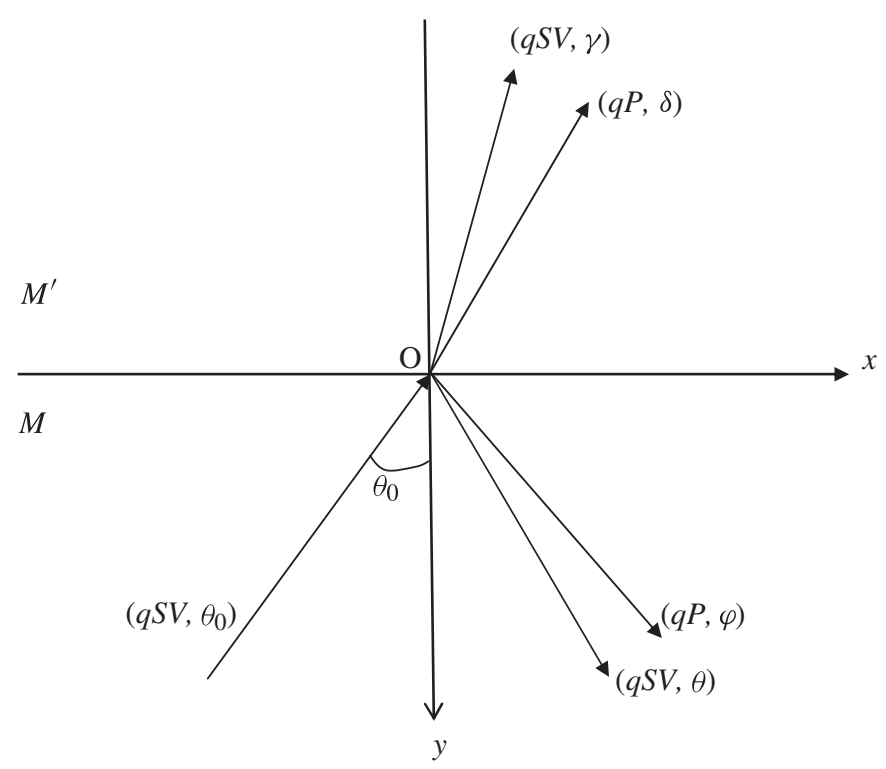

FIGURE 1. Geometry of the problem.

where

$$
E_{1}\left(\theta_{0}\right)=B_{11} \sin ^{2} \theta_{0}+A_{1} \cos ^{2} \theta_{0} \quad \text { and } \quad E_{2}\left(\theta_{0}\right)=B_{22} \cos ^{2} \theta_{0}+A_{2} \sin ^{2} \theta_{0} .
$$

We observe that the velocity of this $q S V$-wave in a pre-stressed elastic medium depends on the angle of propagation. The incident plane $q S V$-wave gives rise to reflected $q S V$ - and $q P$-waves in the half-space $M$ and refracted $q S V$ - and $q P$-waves in $M^{\prime}$. The situation is illustrated in Figure 1.

The total displacement in the half-space $M$ is given by the sum of the displacements due to the incident and reflected waves as

$$
\begin{aligned}
& U=G_{0} \exp \left[\frac{l \omega}{c_{2}}\left\{c_{2} t-\left(x \sin \theta_{0}-y \cos \theta_{0}\right)\right\}\right] \\
&+ G_{1} \exp \left[\frac{l \omega}{c_{2}}\left\{c_{2} t-(x \sin \theta+y \cos \theta)\right\}\right] \\
&+ G_{2} \exp \left[\frac{l \omega}{c_{1}}\left\{c_{1} t-(x \sin \phi+y \cos \phi)\right\}\right], \\
& V=H_{0} \exp \left[\frac{l \omega}{c_{2}}\left\{c_{2} t-\left(x \sin \theta_{0}-y \cos \theta_{0}\right)\right\}\right] \\
&+H_{1} \exp \left[\frac{l \omega}{c_{2}}\left\{c_{2} t-(x \sin \theta+y \cos \theta)\right\}\right] \\
&+H_{2} \exp \left[\frac{l \omega}{c_{1}}\left\{c_{1} t-(x \sin \phi+y \cos \phi)\right\}\right],
\end{aligned}
$$


where $\omega=k c$ is the angular velocity, $\iota=\sqrt{-1}, G_{0}$ and $H_{0}$ are the amplitude constants of the horizontal and vertical components of the displacement due to the incident $q S V$ wave, $G_{1}$ and $H_{1}$ are the corresponding amplitude constants of the reflected $q S V$-wave with phase velocity $c_{2}$ at angle $\theta$, and $G_{2}$ and $H_{2}$ are the amplitude constants of the reflected $q P$-wave with phase velocity $c_{1}$ at angle $\phi$. Sidhu and Singh [17] derived the relation between the amplitude constants of the horizontal and vertical components of the displacement as

$$
G_{0}=F_{0} H_{0}, \quad G_{1}=-F_{1} H_{1}, \quad G_{2}=-F_{2} H_{2},
$$

where

$$
\begin{aligned}
& F_{0}=\frac{A_{3} \sin \theta_{0} \cos \theta_{0}}{E_{1}\left(\theta_{0}\right)-\rho c_{2}^{2}}, \quad F_{1}=\frac{A_{3} \sin \theta \cos \theta}{E_{1}(\theta)-\rho c_{2}^{2}}, \quad F_{2}=\frac{A_{3} \sin \phi \cos \phi}{E_{1}(\phi)-\rho_{1} c_{1}^{2}}, \\
& E_{1}(\theta)=B_{11} \sin ^{2} \theta+A_{1} \cos ^{2} \theta, \quad E_{1}(\phi)=B_{11} \sin ^{2} \phi+A_{1} \cos ^{2} \phi \\
& E_{2}(\theta)=B_{22} \cos ^{2} \theta+A_{2} \sin ^{2} \theta, \quad E_{2}(\phi)=B_{22} \cos ^{2} \phi+A_{2} \sin ^{2} \phi \\
& 2 \rho c_{1}^{2}=E_{1}(\phi)+E_{2}(\phi)+\sqrt{\left\{E_{1}(\phi)-E_{2}(\phi)\right\}^{2}+4 A_{3}^{2} \sin ^{2} \phi \cos ^{2} \phi}, \\
& 2 \rho c_{2}^{2}=E_{1}(\theta)+E_{2}(\theta)-\sqrt{\left\{E_{1}(\theta)-E_{2}(\theta)\right\}^{2}+4 A_{3}^{2} \sin ^{2} \theta \cos ^{2} \theta}
\end{aligned}
$$

Similarly, the total displacement in the half-space $M^{\prime}$ is given by the sum of the displacements due to the refracted waves as

$$
\begin{aligned}
& U^{\prime}=G_{1}^{\prime} \exp \left[\frac{l \omega}{c_{2}^{\prime}}\left\{c_{2}^{\prime} t-(x \sin \gamma-y \cos \gamma)\right\}\right] \\
&+ G_{2}^{\prime} \exp \left[\frac{l \omega}{c_{1}^{\prime}}\left\{c_{1}^{\prime} t-(x \sin \delta-y \cos \delta)\right\}\right], \\
& V^{\prime}=H_{1}^{\prime} \exp \left[\frac{l \omega}{c_{2}^{\prime}}\left\{c_{2}^{\prime} t-(x \sin \gamma-y \cos \gamma)\right\}\right] \\
&+H_{2}^{\prime} \exp \left[\frac{l \omega}{c_{1}^{\prime}}\left\{c_{1}^{\prime} t-(x \sin \delta-y \cos \delta)\right\}\right],
\end{aligned}
$$

where $G_{1}^{\prime}$ and $H_{1}^{\prime}$ are the amplitude constants corresponding to the refracted $q S V$ wave with phase velocity $c_{2}^{\prime}$ at angle $\gamma$, and $G_{2}^{\prime}$ and $H_{2}^{\prime}$ are the amplitude constants of the refracted $q P$-wave with phase velocity $c_{1}^{\prime}$ at angle $\delta$. The relation between the amplitude constants is given by Singh and Tomar [23] as

$$
G_{1}^{\prime}=F_{1}^{\prime} H_{1}^{\prime}, \quad G_{2}^{\prime}=F_{2}^{\prime} H_{2}^{\prime},
$$

where

$$
\begin{aligned}
& F_{1}^{\prime}=\frac{A_{3}^{\prime} \sin \gamma \cos \gamma}{E_{1}^{\prime}(\gamma)-\rho^{\prime} c_{2}^{\prime 2}}, \quad F_{2}^{\prime}=\frac{A_{3}^{\prime} \sin \delta \cos \delta}{E_{1}^{\prime}(\delta)-\rho^{\prime} c_{1}^{\prime 2}}, \\
& E_{1}^{\prime}(\gamma)=B_{11}^{\prime} \sin ^{2} \gamma+A_{1}^{\prime} \cos ^{2} \gamma, \quad E_{1}^{\prime}(\delta)=B_{11}^{\prime} \sin ^{2} \delta+A_{1}^{\prime} \cos ^{2} \delta, \\
& E_{2}^{\prime}(\gamma)=B_{22}^{\prime} \cos ^{2} \gamma+A_{2}^{\prime} \sin ^{2} \gamma, \quad E_{2}^{\prime}(\delta)=B_{22}^{\prime} \cos ^{2} \delta+A_{2}^{\prime} \sin ^{2} \delta,
\end{aligned}
$$




$$
\begin{aligned}
& 2 \rho^{\prime} c_{1}^{\prime 2}=E_{1}^{\prime}(\delta)+E_{2}^{\prime}(\delta)+\sqrt{\left\{E_{1}^{\prime}(\delta)-E_{2}^{\prime}(\delta)\right\}^{2}+4 A_{3}^{\prime 2} \sin ^{2} \delta \cos ^{2} \delta} \\
& 2 \rho^{\prime} c_{2}^{\prime 2}=E_{1}^{\prime}(\gamma)+E_{2}^{\prime}(\gamma)-\sqrt{\left\{E_{1}^{\prime}(\gamma)-E_{2}^{\prime}(\gamma)\right\}^{2}+4 A_{3}^{\prime 2} \sin ^{2} \gamma \cos ^{2} \gamma}
\end{aligned}
$$

The relation between the angle of incidence and the angles of the reflected and refracted waves is given by Snell's law, as by Singh and Tomar [23]:

$$
\frac{\sin \theta_{0}}{c_{2}\left(\theta_{0}\right)}=\frac{\sin \theta}{c_{2}(\theta)}=\frac{\sin \phi}{c_{1}(\phi)}=\frac{\sin \gamma}{c_{2}^{\prime}(\gamma)}=\frac{\sin \delta}{c_{1}^{\prime}(\delta)}=\frac{1}{c_{\mathrm{a}}},
$$

where $c_{\mathrm{a}}$ is the apparent velocity, namely the velocity of propagation of the points of intersection of wave fronts with the plane of separation.

\section{Boundary conditions}

The boundary conditions are the continuity of the displacement components and incremental forces in the half-spaces at the plane interface $y=0$, given by

$$
\begin{gathered}
V=V^{\prime}, \quad U=U^{\prime}, \\
A_{01} \frac{\partial V}{\partial x}+A_{1} \frac{\partial U}{\partial y}=A_{01}^{\prime} \frac{\partial V^{\prime}}{\partial x}+A_{1}^{\prime} \frac{\partial U^{\prime}}{\partial y} \\
B_{1} \frac{\partial U}{\partial x}+B_{22} \frac{\partial V}{\partial y}=B_{1}^{\prime} \frac{\partial U^{\prime}}{\partial x}+B_{22}^{\prime} \frac{\partial V^{\prime}}{\partial y}
\end{gathered}
$$

where $A_{01}=Q-\left(S_{22}+S_{11}\right) / 2, A_{01}^{\prime}=Q^{\prime}-\left(S_{22}^{\prime}+S_{11}^{\prime}\right) / 2, B_{1}=B_{12}+S_{11}$ and $B_{1}^{\prime}=$ $B_{12}^{\prime}+S_{11}^{\prime}$. Using (3.2)-(3.8) in (4.1)-(4.3),

$$
\begin{aligned}
& \frac{H_{1}}{H_{0}}+\frac{H_{2}}{H_{0}}-\frac{H_{1}^{\prime}}{H_{0}}-\frac{H_{2}^{\prime}}{H_{0}}=-1, \\
& F_{1} \frac{H_{1}}{H_{0}}+F_{2} \frac{H_{2}}{H_{0}}+F_{1}^{\prime} \frac{H_{1}^{\prime}}{H_{0}}+F_{2}^{\prime} \frac{H_{2}^{\prime}}{H_{0}}=F_{0}, \\
& a_{31} \frac{H_{1}}{H_{0}}+a_{32} \frac{H_{2}}{H_{0}}+a_{33} \frac{H_{1}^{\prime}}{H_{0}}+a_{34} \frac{H_{2}^{\prime}}{H_{0}}=d_{3}, \\
& a_{41} \frac{H_{1}}{H_{0}}+a_{42} \frac{H_{2}}{H_{0}}+a_{43} \frac{H_{1}^{\prime}}{H_{0}}+a_{44} \frac{H_{2}^{\prime}}{H_{0}}=d_{4},
\end{aligned}
$$

where

$$
\begin{array}{ll}
a_{31}=-A_{01} \sin \theta_{0} / c_{2}+A_{1} F_{1} \cos \theta / c_{2}, & a_{32}=-A_{01} \sin \theta_{0} / c_{2}+A_{1} F_{2} \cos \phi / c_{1}, \\
a_{33}=A_{01}^{\prime} \sin \theta_{0} / c_{2}-A_{1}^{\prime} F_{1}^{\prime} \cos \gamma / c_{2}^{\prime}, & a_{34}=A_{01}^{\prime} \sin \theta_{0} / c_{2}-A_{1}^{\prime} F_{2}^{\prime} \cos \delta / c_{1}^{\prime}, \\
a_{41}=B_{1} F_{1} \sin \theta_{0} / c_{2}-B_{22} \cos \theta / c_{2}, & a_{42}=B_{1} F_{2} \sin \theta_{0} / c_{2}-B_{22} \cos \phi / c_{1}, \\
a_{43}=B_{1}^{\prime} F_{1}^{\prime} \sin \theta_{0} / c_{2}-B_{22}^{\prime} \cos \gamma / c_{2}^{\prime}, & a_{44}=B_{1}^{\prime} F_{2}^{\prime} \sin \theta_{0} / c_{2}-B_{22}^{\prime} \cos \delta / c_{1}^{\prime}, \\
d_{3}=A_{01} \sin \theta_{0} / c_{2}-A_{1} F_{1} \cos \theta_{0} / c_{2}, & d_{4}=B_{1} F_{0} \sin \theta_{0} / c_{2}-B_{22} \cos \theta_{0} / c_{2} .
\end{array}
$$


These equations are used to obtain the amplitude ratios corresponding to the vertical components of the reflected and refracted elastic waves.

\section{Reflection and refraction coefficients}

Applying Cramer's rule for solving a system of linear equations to (4.4)-(4.7), we obtain the amplitude ratios corresponding to the vertical components of the displacement as

$$
\frac{H_{1}}{H_{0}}=\frac{\Delta_{H 1}}{\Delta}, \quad \frac{H_{2}}{H_{0}}=\frac{\Delta_{H 2}}{\Delta}, \quad \frac{H_{1}^{\prime}}{H_{0}}=\frac{\Delta_{H 1}^{\prime}}{\Delta}, \quad \frac{H_{2}^{\prime}}{H_{0}}=\frac{\Delta_{H 2}^{\prime}}{\Delta},
$$

where

$$
\Delta=\left|\begin{array}{cccc}
1 & 1 & -1 & -1 \\
F_{1} & F_{2} & F_{1}^{\prime} & F_{2}^{\prime} \\
a_{31} & a_{32} & a_{33} & a_{34} \\
a_{41} & a_{42} & a_{43} & a_{44}
\end{array}\right| \quad \text { and for } \quad D=\left[\begin{array}{c}
-1 \\
F_{0} \\
d_{3} \\
d_{4}
\end{array}\right]
$$

the quantities $\Delta_{H 1}, \Delta_{H 2}, \Delta_{H 1}^{\prime}$ and $\Delta_{H 2}^{\prime}$ are obtained by replacing the first, second, third and fourth columns of the determinant $\Delta$, respectively, with the column matrix $D$. Putting (3.4) and (3.7) into (5.1), we find the amplitude ratios corresponding to the horizontal components of the displacement of the reflected and refracted elastic waves:

$$
\frac{G_{1}}{G_{0}}=-\frac{F_{1}}{F_{0}} \frac{\Delta_{H 1}}{\Delta}, \quad \frac{G_{2}}{G_{0}}=-\frac{F_{2}}{F_{0}} \frac{\Delta_{H 2}}{\Delta}, \quad \frac{G_{1}^{\prime}}{G_{0}}=\frac{F_{1}^{\prime}}{F_{0}} \frac{\Delta_{H 1}^{\prime}}{\Delta}, \quad \frac{G_{2}^{\prime}}{G_{0}}=\frac{F_{2}^{\prime}}{F_{0}} \frac{\Delta_{H 2}^{\prime}}{\Delta} .
$$

The amplitude of the incident $q S V$-wave is given by $\sqrt{H_{0}^{2}+G_{0}^{2}}=\sqrt{1+F_{0}^{2}} H_{0}$, and the amplitudes of the reflected $q S V$ - and $q P$-waves are respectively given by

$$
\sqrt{H_{1}^{2}+G_{1}^{2}}=\sqrt{1+F_{1}^{2}} H_{1} \text { and } \sqrt{H_{2}^{2}+G_{2}^{2}}=\sqrt{1+F_{2}^{2}} H_{2} .
$$

The reflection coefficients corresponding to reflected $q S V$ - and $q P$-waves are respectively denoted by $R_{s s}$ and $R_{s p}$, and are given by

$$
R_{s s}=\sqrt{\frac{1+F_{1}^{2}}{1+F_{0}^{2}}} \frac{\Delta_{H 1}}{\Delta}, \quad R_{s p}=\sqrt{\frac{1+F_{2}^{2}}{1+F_{0}^{2}}} \frac{\Delta_{H 2}}{\Delta} .
$$

Similarly, the refraction coefficients corresponding to the refracted $q S V$ - and $q P$ waves are

$$
r_{s s}=\sqrt{\frac{1+F_{1}^{\prime 2}}{1+F_{0}^{2}}} \frac{\Delta_{H 1}^{\prime}}{\Delta}, \quad r_{s p}=\sqrt{\frac{1+F_{2}^{\prime 2}}{1+F_{0}^{2}}} \frac{\Delta_{H 2}^{\prime}}{\Delta} .
$$

We observe that these coefficients are functions of the angle of incidence and the incremental elastic constants and initial stresses of the pre-stressed elastic half-spaces. The effect of the initial stresses on these reflection and refraction coefficients is discussed for a particular model in Section 6. 
5.1. Particular case I If the half-space $M^{\prime}$ reduces to a homogeneous isotropic elastic half-space, the problem reduces to the reflection and refraction of elastic waves for incident $q S V$-waves at a plane interface between elastic/pre-stressed elastic halfspaces. In this case, $B_{11}^{\prime}=B_{22}^{\prime}=\lambda^{\prime}+2 \mu^{\prime}, B_{12}^{\prime}=B_{21}^{\prime}=B_{1}^{\prime}=\lambda^{\prime}, A_{1}^{\prime}=A_{2}^{\prime}=Q^{\prime}=A_{01}^{\prime}=$ $\mu^{\prime}, A_{3}^{\prime}=\lambda^{\prime}+\mu^{\prime}$ and $S_{11}^{\prime}=S_{22}^{\prime}=0$ in $M^{\prime}$, and the phase velocities of the elastic waves are given by

$$
c_{2}^{\prime}=\sqrt{\frac{\mu^{\prime}}{\rho^{\prime}}}, \quad c_{1}^{\prime}=\sqrt{\frac{\lambda^{\prime}+2 \mu^{\prime}}{\rho^{\prime}}}, \quad F_{1}^{\prime}=\cot \gamma, \quad F_{2}^{\prime}=-\tan \delta .
$$

The reflection and refraction coefficients corresponding to the reflected and refracted elastic waves are given by (5.2) and (5.3) with the following modified values of $a_{33}$, $a_{34}, a_{43}$ and $a_{44}$ :

$$
\begin{aligned}
& a_{33}=\left(\frac{\sin \theta_{0}}{c_{2}}-\frac{\cot \gamma \cos \gamma}{c_{2}^{\prime}}\right) \mu^{\prime}, \quad a_{34}=\left(\frac{\sin \theta_{0}}{c_{2}}+\frac{\tan \delta \cos \delta}{c_{1}^{\prime}}\right) \mu^{\prime}, \\
& a_{43}=\lambda^{\prime} \frac{\cot \gamma \sin \theta_{0}}{c_{2}}-\left(\lambda^{\prime}+2 \mu^{\prime}\right) \frac{\cos \gamma}{c_{2}^{\prime}}, \quad a_{44}=-\lambda^{\prime} \frac{\tan \delta \sin \theta_{0}}{c_{2}}-\left(\lambda^{\prime}+2 \mu^{\prime}\right) \frac{\cos \delta}{c_{1}^{\prime}} .
\end{aligned}
$$

These coefficients depend on the angle of incidence and the elastic parameters, initial stresses and incremental elastic constants.

5.2. Particular case II If the elastic half-space $M^{\prime}$ is absent, the problem reduces to the reflection of elastic waves for the incident $q S V$-wave at the plane free boundary in the homogeneous pre-stressed elastic half-space $M$. In this case, the quantities corresponding to $M^{\prime}$ are equal to zero. If we assume that the angle of incidence of the $q S V$-wave is equal to the angle of the reflected $q S V$-wave, then $a_{31}=-d_{3}, F_{0}=F_{1}$ and $a_{41}=d_{4}$, and the reflection coefficients of the reflected $q S V$ - and $q P$-waves at the plane free surface in the pre-stressed elastic half-space are given by (5.2) with the modified values

$$
\Delta=a_{32} a_{41}-a_{42} a_{31}, \quad \Delta_{H 1}=a_{32} a_{41}+a_{42} a_{31}, \quad \Delta_{H 2}=-2 a_{31} a_{41} .
$$

These coefficients match exactly with the results of Sidhu and Singh [17] for the corresponding problem.

\section{Numerical results and discussion}

In order to see the effect of the initial stresses on the propagation of $q S V$-waves, a FORTRAN program was used to compute the reflection and refraction coefficients of the reflected and refracted elastic waves. The following parameters were used. In the half-space $M$ :

$$
\begin{gathered}
B_{11}=6.5 \times 10^{11} \mathrm{~N} \mathrm{~m}^{-2}, \quad B_{22}=10.99 \times 10^{12} \mathrm{~N} \mathrm{~m}^{-2}, \\
B_{21}=1.56 \times 10^{11} \mathrm{~N} \mathrm{~m}^{-2}, \quad Q=1.22 \times 10^{11} \mathrm{~N} \mathrm{~m}^{2} \quad \text { and } \rho=1800 \mathrm{~kg} \mathrm{~m}^{-3} .
\end{gathered}
$$


In the half-space $M^{\prime}$ :

$$
\begin{gathered}
B_{11}^{\prime}=5.344 \times 10^{11} \mathrm{~N} \mathrm{~m}^{-2}, \quad B_{22}^{\prime}=2.127 \times 10^{11} \mathrm{~N} \mathrm{~m}^{-2}, \\
B_{21}^{\prime}=1.52 \times 10^{11} \mathrm{~N} \mathrm{~m}^{-2}, \quad Q^{\prime}=1.58 \times 10^{11} \mathrm{~N} \mathrm{~m}^{-2} \quad \text { and } \quad \rho^{\prime}=2900 \mathrm{~kg} \mathrm{~m}^{-3} .
\end{gathered}
$$

The angles $\theta$ for the reflected $q S V$-wave, $\phi$ for the reflected $q P$-wave, $\gamma$ for the refracted $q S V$-wave and $\delta$ for the refracted $q P$-wave depend on the angle of incidence $\theta_{0}$. These angles may be obtained by using Snell's law given in (3.8), in which the dimensionless apparent velocity is $\bar{c}$, given by $\bar{c}=c_{2} / p_{2} \beta$. Using this apparent velocity for the reflected angles in the half-space $M$ in (3.1),

$$
\bar{c}^{4}-\left(\bar{E}_{1}+\bar{E}_{2}\right) \bar{c}^{2}+\bar{E}_{1} \bar{E}_{2}-\bar{A}_{3}^{2} p^{2}=0,
$$

where $p=p_{3} / p_{2},\left(p_{2}, p_{3}\right)=\left(\sin \theta_{0}, \cos \theta_{0}\right)$ and

$$
\bar{E}_{1}=\frac{E_{1}}{B_{11} p_{2}^{2}}, \quad \bar{E}_{2}=\frac{E_{2}}{B_{11} p_{2}^{2}}, \quad \bar{A}_{3}=\frac{A_{3}}{B_{11}}, \quad \beta=\sqrt{\frac{B_{11}}{\rho}} .
$$

Equation (6.1) gives two positive roots $\bar{c}$; the smaller one corresponds to the reflected $q S V$-wave and the larger to the reflected $q P$-wave. For a given value of $\bar{c}$, there are two positive values of $\theta_{0}$, which correspond to the reflected angles $\theta$ and $\phi$. Inserting the above values of $\bar{E}_{1}, \bar{E}_{2}$ and $\bar{A}_{3}$ into (6.1) gives

$$
g_{0} p^{4}+g_{2} p^{2}+g_{4}=0,
$$

where

$$
\begin{aligned}
g_{0}=\frac{A_{1}}{B_{11}} \frac{A_{2}}{B_{11}}, \quad g_{2} & =\frac{A_{1}}{B_{11}} \frac{B_{22}}{B_{11}}+\frac{A_{2}}{B_{11}}-\frac{A_{3}^{2}}{B_{11}^{2}}-\left(\frac{A_{1}}{B_{11}}+\frac{A_{2}}{B_{11}}\right) \bar{c}^{2}, \\
g_{4} & =\bar{c}^{4}-\left(1+\frac{B_{22}}{B_{11}}\right) \bar{c}^{2}+\frac{B_{22}}{B_{11}} .
\end{aligned}
$$

Let us transform the roots of the above equation as $q=1 / p=p_{2} / p_{3}$, so that

$$
g_{4} q^{4}+g_{2} q^{2}+g_{0}=0 .
$$

This equation has two positive roots $q$. The smaller positive root, denoted by $q_{2}$, corresponds to the reflected $q S V$-wave, and the larger positive root, $q_{1}$, to the reflected $q P$-wave. We have

$$
\theta=\tan ^{-1}\left(q_{2}\right), \quad \phi=\tan ^{-1}\left(q_{1}\right) .
$$

Similarly, we obtain the angles corresponding to the refracted $q S V$ - and $q P$-waves in the half-space $M^{\prime}$ as

$$
\gamma=\tan ^{-1}\left(q_{2}^{\prime}\right), \quad \delta=\tan ^{-1}\left(q_{1}^{\prime}\right) .
$$

Using these angles of propagation, the reflection and refraction coefficients are computed numerically. The variation of the coefficients corresponding to the reflected 

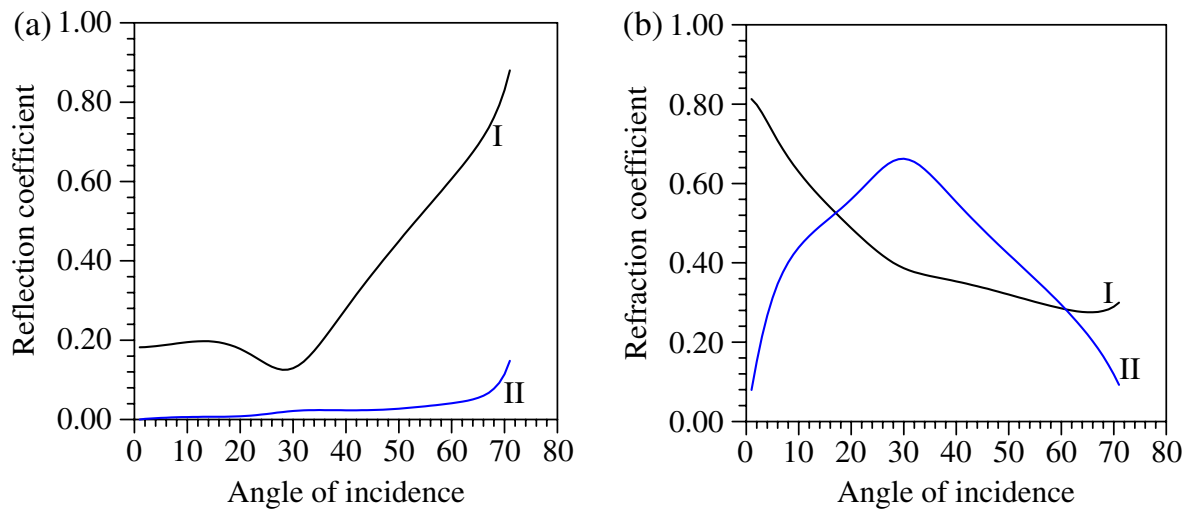

FIGURE 2. (a) Variation of $R_{s s}$ (curve I) and $R_{s p}$ (curve II) with $\theta_{0}$ without initial stresses. (b) Variation of $r_{s s}$ (curve I) and $r_{s p}$ (curve II) with $\theta_{0}$ without initial stresses.

and refracted $q S V$ - and $q P$-waves with respect to the angle of incidence, $\theta_{0}$, is shown in Figure 2. The variation of the reflected and refracted coefficients for different values of the initial stresses is depicted in Figure 3.

In Figure 2(a), curve I shows that the reflection coefficient $R_{s S}$ corresponding to the reflected $q S V$-wave increases up to $\theta_{0}=13^{\circ}$, decreases to its minimum value at $\theta_{0}=28^{\circ}$ and then increases. Curve II shows that the reflection coefficient $R_{s p}$ corresponding to the reflected $q P$-wave increases with increasing angle of incidence. Curve I in Figure 2(b) shows that the refraction coefficient $r_{s s}$ corresponding to the refracted $q S V$-wave starts from its maximum value at normal incidence, decreases up to $\theta_{0}=68^{\circ}$ and increases thereafter. Curve II shows that the refraction coefficient $r_{s p}$ corresponding to the refracted $q P$-wave increases to its maximum value at $\theta_{0}=30^{\circ}$ and then decreases. We observe that all four coefficients have critical values at $\theta_{0}=71^{\circ}$ due to the condition $\theta=\sin ^{-1}\left[c_{2}(\phi) / c_{2}(\pi / 2)\right]$ for the reflected $q S V$-wave and similar conditions for the reflected $q P$-wave and refracted $q S V$ - and $q P$-waves. At this particular angle of incidence, all the angles corresponding to the reflected and refracted waves propagate at the grazing angle of propagation. As a result of this limitation, there are no reflection or refraction coefficients after the angle of incidence $\theta_{0}=71^{\circ}$.

To see the effect of the initial stresses on the reflection and refraction coefficients, the following initial stresses are taken in Figure 3. Curve I: $S_{11}=S_{11}^{\prime}=1.0 \times$ $10^{10} \mathrm{~N} \mathrm{~m}^{-2}, S_{22}=S_{22}^{\prime}=0$; curve II: $S_{22}=S_{22}^{\prime}=1.0 \times 10^{10} \mathrm{~N} \mathrm{~m}^{-2}, S_{11}=S_{11}^{\prime}=0$; curve III: $S_{11}=S_{22}=S_{11}^{\prime}=S_{22}^{\prime}=1.5 \times 10^{10} \mathrm{~N} \mathrm{~m}^{-2}$. Figure 3(a) shows that $R_{s s}$ decreases with initial stresses. The maximum effect of the initial stresses is observed near $\theta_{0}=30^{\circ}$, and the minimum near the normal angle of incidence. Figure 3(b) shows that the effect of the initial stresses on $R_{s p}$ is maximal near $\theta_{0}=30^{\circ}$ and minimal near normal incidence. In Figure 3(c), the effect of the initial stresses on $r_{s s}$ is observed to be maximal near normal incidence and minimal at $\theta_{0}=66^{\circ}$. Figure $3(\mathrm{~d})$ shows that the 

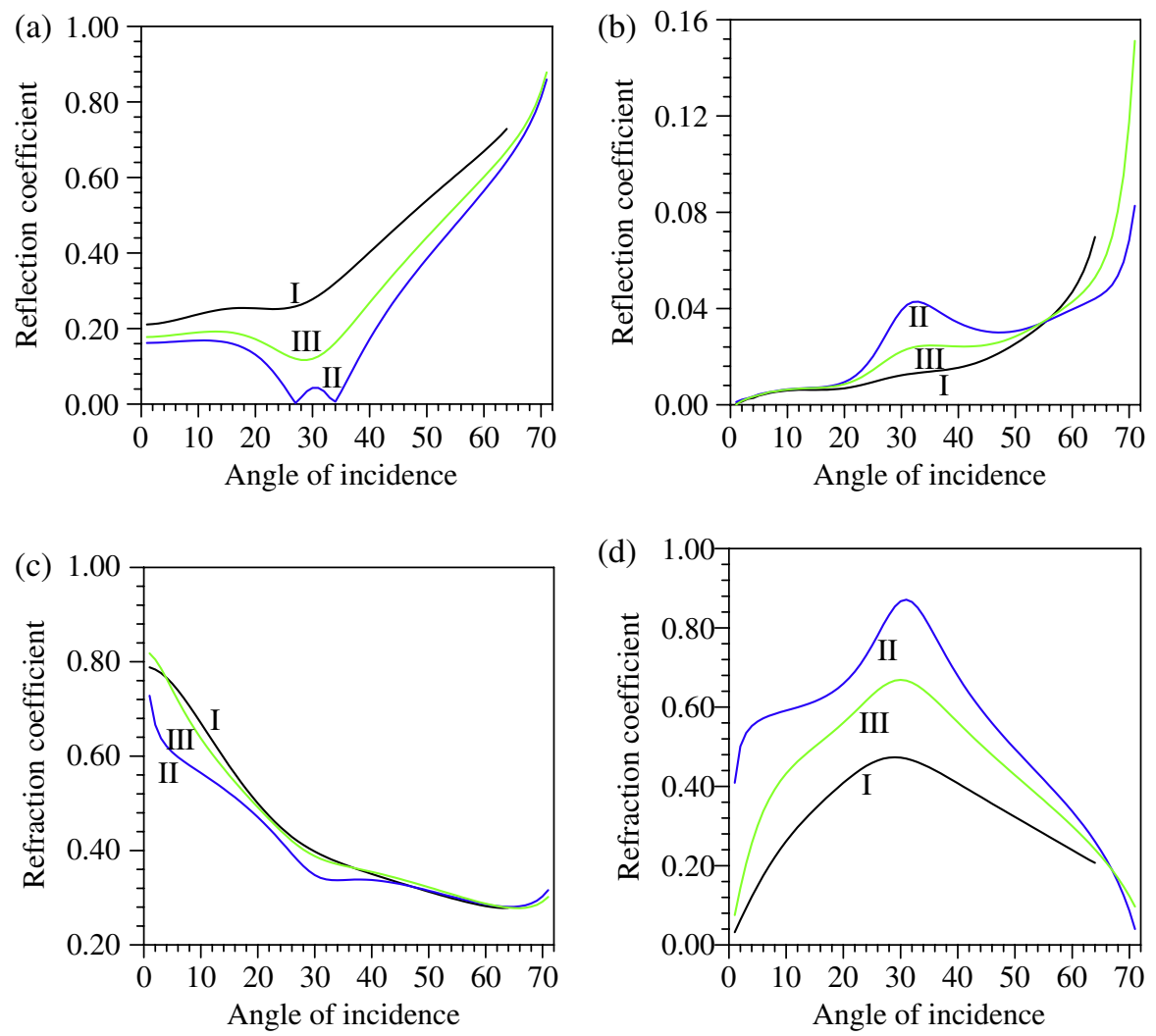

FIGURE 3. Variation of (a) $R_{s s}$, (b) $R_{s p}$, (c) $r_{s s}$, (d) $r_{s p}$ with $\theta_{0}$ with initial stresses $S_{11}=S_{22}=1$, $S_{11}^{\prime}=S_{22}^{\prime}=0$ (curve I); $S_{11}=S_{22}=0, S_{11}^{\prime}=S_{22}^{\prime}=1$ (curve II); $S_{11}=S_{22}=S_{11}^{\prime}=S_{22}^{\prime}=1.5$ (curve III).

effect of the initial stresses on $r_{s p}$ is greater at lower values of the angle of incidence, with the minimal effect observed near $\theta_{0}=66^{\circ}$.

\section{Conclusion}

The effect of initial stresses on the propagation of incident $q S V$-waves at the plane interface between two dissimilar homogeneous pre-stressed elastic half-spaces has been investigated. The reflection and refraction coefficients corresponding to the reflected and refracted $q S V$ - and $q P$-waves have been presented in closed form. In order to discuss the effect of the initial stresses, the reflection and refraction coefficients have been computed numerically for a particular model. We conclude that:

(i) the reflection and refraction coefficients are functions of the initial stresses, incremental elastic coefficients and angle of incidence;

(ii) the results of Sidhu and Singh [17] are recovered from our analysis;

(iii) all the coefficients have critical angle of incidence $\theta_{0}=71^{\circ}$; 
(iv) the maximum effect of the initial stresses on the reflection coefficients occurs near $\theta_{0}=30^{\circ}$;

(v) the maximum effect of the initial stresses on the refraction coefficient $r_{s s}$ is observed near the normal angle of incidence.

\section{Acknowledgements}

The author acknowledges the Department of Science \& Technology (DST), New Delhi, India for financial support through Grant No. SR/FTP/MS-017/2010. The author is also thankful to Dr. R. K. Bhattachary for his valuable suggestion to improve the manuscript.

\section{References}

[1] A. N. Abd-alla and F. A. Alsheikh, "Reflection and refraction of plane quasilongitudinal waves at an interface of two piezoelectric media under initial stresses", Arch. Appl. Mech. 79 (2009) 843-857; doi:10.1007/s00419-008-0257-y.

[2] H. K. Acharya, "Reflection from the free surface of an inhomogeneous media", Bull. Seismol. Soc. Amer. 60 (1970) 1101-1104.

[3] J. D. Achenbach, Wave propagation in elastic solids (North-Holland, New York, 1973).

[4] S. Yu. Babich, "Propagation of surface waves in a prestressed medium", Int. Appl. Mech. 14 (1978) 1004-1007; doi:10.1007/BF00885757.

[5] M. A. Biot, "The influence of initial stress on elastic waves", J. Appl. Phys. 11 (1940) 522-530; doi:10.1063/1.1712807.

[6] M. A. Biot, Mechanics of incremental deformations (John Wiley, New York, 1965).

[7] A. Chattopadhyay, S. Bose and M. Chakraborty, "Reflection of elastic waves under initial stress at a free surface: $P$ and $S V$ motion", J. Acoust. Soc. Am. 72 (1982) 255-263; doi:10.1121/1.387987.

[8] M. A. Dowaikh and R. W. Ogden, "Interfacial waves and deformations in pre-stressed elastic media”, Proc. R. Soc. Lond. A 433 (1991) 313-328; doi:10.1098/rspa.1991.0050.

[9] J. Du, X. Jin and J. Wang, "Love wave propagation in layered magneto-electro-elastic structures with initial stress", Acta Mech. 192 (2007) 169-189; doi:10.1007/s00707-006-0435-3.

[10] N. Garg, "Existence of longitudinal waves in pre-stressed anisotropic elastic medium", J. Earth Syst. Sci. 118 (2009) 677-687; doi:10.1007/s12040-009-0053-2.

[11] A. N. Guz, "Elastic waves in bodies with initial (residual) stresses", Int. Appl. Mech. 38 (2002) 23-59; doi:10.1023/A:1015379824503.

[12] P. Khurana and A. K. Vashisth, "Love wave propagation in a pre-stressed medium", Indian J. Pure Appl. Math. 32 (2001) 1201-1207.

[13] A. N. Norris, "Propagation of plane waves in a pre-stressed elastic medium", J. Acoust. Soc. Am. 74 (1983) 1642-1643; doi:10.1121/1.390131.

[14] R. W. Ogden and D. A. Sotiropoulos, "On interfacial waves in pre-stressed layered incompressible elastic solids", Proc. R. Soc. Lond. A 450 (1995) 319-341; doi:10.1098/rspa.1995.0087.

[15] M. D. Sharma, "Effect of initial stress on reflection at the free surface of anisotropic elastic medium", J. Earth Syst. Sci. 116 (2007) 537-551; doi:10.1007/s12040-007-0049-8.

[16] R. S. Sidhu and S. J. Singh, "Comments on reflection of elastic waves under initial stress at a free surface: $P$ and $S V$ motion (J. Acoust. Soc. Am. 72, 255-263 (1982))", J. Acoust. Soc. Am. 74 (1983) 1640-1642; doi:10.1121/1.390130.

[17] R. S. Sidhu and S. J. Singh, "Reflection of $P$ and $S V$ waves at the free surface of a prestressed elastic half-space", J. Acoust. Soc. Am. 76 (1984) 594-598; doi:10.1121/1.391155.

[18] R. S. Sidhu and S. J. Singh, "On the existence of quasi $S$ waves in a prestressed elastic solid", J. Acoust. Soc. Am. 78 (1985) 803-804; doi:10.1121/1.392400. 
[19] B. Singh, "Wave propagation in a prestressed piezoelectric half-space", Acta Mech. 211 (2010) 337-344; doi:10.1007/s00707-009-0234-8.

[20] S. J. Singh and S. Khurana, "Reflection and transmission of $P$ and $S V$ waves at the interface between monoclinic elastic half-spaces", Proc. Natl. Acad. Sci. India A 71 (2001) 305-319.

[21] S. J. Singh and S. Khurana, "Reflection of $P$ and $S V$ waves at the free surface of a monoclinic elastic half-space", Proc. Indian Acad. Sci. 111 (2002) 401-412.

[22] I. Singh, D. K. Madan and M. Gupta, "Propagation of elastic waves in prestressed media", J. Appl. Math. (2010) Article ID 817680; doi:10.1155/2010/817680.

[23] S. S. Singh and S. K. Tomar, " $q P$-wave at a corrugated interface between two dissimilar prestressed elastic half-spaces", J. Sound Vib. 317 (2008) 687-708; doi:10.1016/j.jsv.2008.03.036.

[24] T. C. T. Ting, "Longitudinal and transverse waves in anisotropic elastic materials", Acta Mech. 185 (2006) 147-164; doi:10.1007/s00707-006-0333-8. 